\title{
Fuzzy Logic Based Path Stability Forecasting Scheme for Improving Data Dissemination in MANETs
}

\author{
Calarany C., Manoharan R.
}

\begin{abstract}
Path stability of the mobile nodes in MANET plays a vital role in effective data dissemination as it depends on factors such as mobility, energy, signal strength. Several studies reveal that the prediction of path stability might provide solutions thereby routing performance can be increased. In most of the protocols route selection is based on metrics namely hop count, energy, etc. The metric namely mobility factor "MF" is used in some of the protocols. These protocols include nodes with less energy or nodes with high mobility which results in loss of path in a short period of time. Since it preserves the neighbor's history, more control overhead and maintenance complexity exist. Hence, a new metric namely Active Interactive new Neighbor Rate (AINR) has been considered for optimum path selection. In scenarios of path loss, there is an immediate need for alternative paths for continuous data transfer. From literature it is evident that fuzzy logic is more significant in exploring different possible states under path stability determination. Hence a new prediction mechanism based on fuzzy logic has been proposed by considering the Residual Energy (RE), Hop count (Hop) and proposed metric Active Interactive new Neighbor Rate (AINR) as the factors for the prediction of the optimal path. This prediction mechanism is leveraged in MANET scenarios where alternate paths should be available on hand in situations such as battlefield and natural disaster. From the simulation, it has been proved that fuzzy logic prediction model provides better results in terms of various performance metrics such as Throughput, Packet delivery ratio, End-to-end delay, Energy consumption and routing overhead than the existing protocols.
\end{abstract}

Keywords: Active interactive new Neighbor rate, Energy, Fuzzy logic, Mobile Ad hoc Networks, Path Stability.

\section{INTRODUCTION}

Infrastructure less network with dynamic topology makes routing in mobile ad hoc networks as a difficult task. All the nodes are highly mobile in nature which results in a dynamic topology. But they act both as a host as well as a router. The nodes in the network can organize themselves when they are within the transmission range. Due to this transmission limitation, the nodes may not connect to the destination directly. Selection of the intermediate node has a

Revised Manuscript Received on February 05, 2020.

* Correspondence Author

C. Calarany*, Assistant Professor, Tagore Govt. Arts and Science College, Pondicherry, India.

R. Manoharan, Professor, Department of Computer Science and Engineering, Pondicherry Engineering College, Puducherry, India.

(c) The Authors. Published by Blue Eyes Intelligence Engineering and Sciences Publication (BEIESP). This is an open access article under the CC BY-NC-ND license (http://creativecommons.org/licenses/by-nc-nd/4.0/) great impact on path stability. There are many influential factors which affects the stability of the path such as mobility, battery power of the node, Received signal strength, security, fault tolerance and so on. In the applications such as military tactical networks, emergency networks, etc. connectivity is an essential factor. Many routing protocols have been proposed to solve this issue. However, the hop count metric [1] is used in many of them to select the shortest path which includes lossy links or slow nodes and hence results in poor performance in data transmission. Hence, a new metric, namely Average Encounter Rate [2] has been proposed to predict the node mobility. Multi objective routing protocols provide more better results than single objective or bi-objective protocols [3]. To avoid the packet loss due to the breakage in the path and to minimize discovery of new routes latency, multipath routing is used. In order to predict the path before it expires either due to node's energy depletion or node mobility, a prediction technique is essential. It has been found that there are many time series prediction techniques such as Bayesian theorem, Markov chain, grey prediction model, Fuzzy logic etc. to predict the stability of the path [19-20]. In this paper, fuzzy logic is used to predict the path stability for effective data transmission. In this work, section II analyses about the related work, section III about the proposed work, section IV algorithm, section V simulation results and evaluation and in section VI conclusion and future enhancements.

\section{RELATED WORK}

The existing problems in MANET such as the available energy of the node after the consumption in terms of transmission power, receiving power, over hearing power, idle power, link breakage due to node mobility makes data transmission as a challenging task. Most of the researchers are dealing with the issues to identify the optimal route, maintenance of the route etc. In the following section idea of the work done in optimization of energy and stability of the nodes along the path in data transmission in MANET.

In Paper [2] a new metric namely Average Encounter Rate (AER) is used instead of hop count to find a reliable path. This metric identifies nodes which has lesser in mobility or which lies in lesser dense area. These are not considered while hop count is used as a metric. During the selection of optimal node, $\mathrm{AER}^{2}$ value has been taken instead of AER to minimize the least mean square error. However, energy is not included in this paper. In paper [3] a bi-objective optimization model is used to find the link stability and energy of the node. Single path is determined for data transmission.

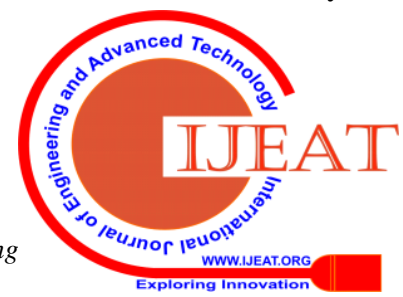


Since path stability is not considered when there is a path loss it requires more computation time to find the alternate path. In paper [4] the congestion is taken as a parameter for energy consumption. According to the author, in many existing energy efficient routing protocols, idle power and sleep power are considered for energy consumption. However, these techniques are not providing more accurate results since congestion is not considered. The nodes are classified based on their current energy level using fuzzy logic. Then load distribution for the rich energy nodes is carried on minimizing the consumption of energy. MAC layer retransmission has been done for local level traffic. However, the node mobility has not been taken into account. In paper [5], a fuzzy based controller has been used for path selection. The parameters used for path selection are length of the queue, average mobility and the distance between the two mobile nodes. The energy factor is not included for path selection. In paper [6] Path selection is based on the parameters namely delay, hop count, signal strength, congestion control and radio metric cost. This protocol uses wireless mesh path protocol (HWMP), which is a combination of both proactive and reactive protocol. However, the energy is not considered.

In paper [7] the author proposed a new routing protocol for energy reduction in MANET. The communication is done by changing the recoil off time depends on their geographical position which in turn reduces the number of retransmissions. Hence the lifetime of the network has been improved. The routing overhead is reduced by maintaining the routes in the local level. Loss of packets in the network has been decreased by forecasting lifetime of the link between the nodes. There are three parts: first one is the optimal route finding, second one is amalgamation in the remaining energy and the third one is the distance of the nodes in the path from the source and destination line. Node mobility is not considered in this paper. In paper [8] a new protocol namely 'Ad hoc on demand multipath routing with lifetime maximization (AOMR-LM)' has been proposed in which residual energy of the node is used for path selection. Two thresholds are used to classify the nodes, namely energy threshold Beta and coefficient Alpha. The selection of the optimal path is based on path class, the class in which the nodes of the path belongs. The nodes are classified as low energy, middle energy and high energy nodes. Path selection is based on high energy nodes. If high energy node is not available then the protocol will select the next higher energy node. Due to multipath selection load balancing is attained and hence network lifetime has been increased. However, the node mobility is not considered in this work.

In paper [9] the author proposed an energy efficient stable routing in which various link related metrics like "link expiration time (LET), probabilistic reliable time of the link (PLRT), link packet error rate (LPER), link received signal strength (LRSS) and remaining batter power (RBP)" are considered as input parameters. Fuzzy logic is used to calculate the probability for the route selection. In the paper [10] the author propounded the relationship between the end to end delay, previous delay and path length. A regression equation is obtained for path length and end to end delay. By using fuzzy time series and regression equation current delay is predicted. The various mobility patterns are taken as input and various prediction techniques such as RMSE, IOE are used for prediction. However, the energy factor is not considered for routing.

In paper [11] the author proposed a new fuzzy logic-based routing protocol namely FBORP to select the optimal path based on the bandwidth, mobility and the residual energy of the nodes. It is proven that the protocol performs well in a high mobility scenario when compared to the benchmark AODV routing protocol. In this protocol based on the weightage given to the parameters different routes are selected. In paper [12] the author proposed a fuzzy based multicasting routing protocol. The Fuzzy rule base depends upon the number of hop counts, sent controlled packets and the energies of the nodes on the routes. The enhancement of FMAR protocol was implemented to quickly maintain and repair the routes with the dynamic lifetime of the routing table before they crashed. In Paper [13] Fuzzy based protocol Fuzzy-AODV is used in which the parameters used are hop count, energy and mobility and the path stability is found using crisp value. However, it provides only single optimal path. In papers [14] the author proposed load balancing ad hoc on-demand multipath distance vector (LBAOMDV) protocol. The energy of the node and channel bandwidth are taken as parameters for load balancing which results in effective data transmission through multiple paths. However, the mobility of the node is not considered for path selection.

In FCMQR protocol [15] path selection is based on end-to-end delay, number of intermediate nodes and bandwidth. Nevertheless, the energy and mobility of the nodes are not considered. In paper [16] The author proposed DFES-AODV protocol in which the best path selection is based on fuzzy logic system in which the parameters used are residual battery power and energy drain rate. In paper [17] The author proposed IAOMDV-F protocol in which the best path selection is based on queue length, distance between the neighbor nodes and mobility. Then it uses path selection index to select the best path. However, the energy of the node is not considered. In paper [18] a new multipath FMRM protocol has been designed in which the fuzzy controllers are used to reduce the route reformation. In paper [19] path stability prediction is based on grey prediction model namely GM $(1,1)$ with energy and active interactive rate as parameters. In paper [20] the path stability prediction has been done based on Markov chain prediction model in which energy is used as parameter to find the stability of the path in each time slot. However, the node mobility is not considered.

From the literature survey it has been found that the mobility prediction of the nodes based on AINR and residual energy using Fuzzy logic has not been done so far.

Published By:
Blue Eyes Intelligence Engineering

\& Sciences Publication 


\section{PROPOSED WORK}

Initially, the path stability of each path is estimated for facilitating reliable data dissemination between the source and destination through five significant steps namely i) Estimation of Active Interactive new Neighbor Rate, ii) Identification of available energy of the path, iii) Computation of hop count iv) Normalized multi-objective optimization, v) Path stability determination and vi) Forecasting of path stability using fuzzy logic. This Fuzzy based Path Stability Forecasting Scheme (FPSFS) integrates the characteristic functionality of ideal solution techniques and fuzzy for forecasting and deciding the stability of the path which can be appropriately used for reliable data routing. The optimal path for data dissemination is chosen based on ideal solution technique by using fuzzy logic for each factor that contribute towards the stability of the path under routing. This ideal solution approach of generating fuzzy aims in investigating the positive and negative influence of each factor that could be derived during the presence and absence of any possible imposable constraints. Finally, the forecasting of reliable path for data routing is achieved depending on the analysis of feasible alternative paths in routing.

\section{A. Estimation of Active Interactive new Neighbor Rate}

In FPSFS, AINR is used for determining the mobility of the node that could be optimally used for data routing. To estimate the mobility of node, appropriate knowledge about the number of new neighbor nodes of each interacting mobile nodes under routing must be determined. AINR refers to the total number of new neighbors that has direct interaction with each specific mobile node under activity in an interval of time $\mathrm{T}[\mathrm{t}-1, \mathrm{t}]$. This count is extracted using periodic broadcasting of hello packets that determine the degree of connectivity which pertains to the exact information of new neighbors under robust connectivity. Each node store and designates the information regarding the set of new neighbors represented using " 1 ".

$$
\operatorname{AINR}=|N i| / T
$$

where $|N i|$ represent the set of new neighbors interacting with a mobile node i.e. Encounters in an interval of time T. The neighbor set of each and every node is periodically refreshed and stored in all the mobile nodes for decision analysis. For Instance, if a mobile node ' $A$ ' is intractable with mobile nodes at time ' $t-1$ ' is $\{B, C, E, F\}$ and ' $t$ ' is $\{B, G, H\}$ then the value of AINR is 2 since $B$ resides there at time ' $t-1$ ' it is not counted twice. When the value of AINR is low then it implies that either the mobility of the node is low since it resides in the same place or the density may be low. PAINR for the path is given by " 2 ".

$$
\text { PAINR }=\sum_{i=1}^{m}\left(\text { AINR }_{i}-\text { MAINR }^{2}\right.
$$

\section{B. Identification of available energy of the node}

Further, energy of the mobile node under interaction in turn contributing towards the energy of the link acts as another factor that potentially influences the stability of the network. The energy of the mobile nodes that establishes a connectivity node is also collected like the AINR parameter of the network which is specific for each participating node under activity. The energy required for transmitting a packet from source node ' $i$ ' is given by " 3 ".

$E_{t x}(p, i)=i . v . t_{p}$

where $i, v$ and $t_{p}$ represents the current (i), voltage (v) and time $\left(t_{p}\right)$ that is essential for transmitting a packet in the network. Further, the total amount of energy required for transmitting a packet from sender node ' $i$ ' to another node ' $j$ ' is computed based on " 4 ".

$E(p, i)=E_{t x}(p, i)+E_{r x}(p, j)+(H-1) E_{0}(p, 0)$

where, $E_{t x}, E_{r x}, E_{o}$ refers to the amount of energy spent for transmitting a packet from sender node ' $i$ ', the energy required for reception of a packet at node ' $j$ ' and energy spent for overhearing the packet by neighbor node respectively. The value of ' $H$ ' highlights the average number of neighbor nodes, which are closely located in the communication range from node i to node $\mathrm{j}$. It is noted that the energy spent on overhearing the packet is considerably very high in the case of dense network.

Furthermore, the drain rate of a mobile node calculated using exponential weighted moving average method for a time interval ' $T$ ' by averaging the amount of energy consumption and energy dissipation estimated during the same time period ' $T$ ' is represented based on " 5 ".

$$
E_{D r(i, t)}=\mathrm{y}_{D r(i, t-1)}+(1-\mathrm{y}) E_{D r(i, t)}
$$

where, ' $\gamma$ ' is the exponentially distributed mean parameter that takes a value between 0 and $1 . E_{\operatorname{Dr}(i, t-1)}$ and $E_{\operatorname{Dr}(i, t)}$ represents the energy drain rate of a mobile node at time $(\mathrm{t}-1)$ and $\mathrm{t}$ of the node respectively. Then, the residual energy of the node $E_{R e(i)}$ is determined based on " 6 ".

$$
E_{\operatorname{Re}(i)}=E_{\operatorname{Or}(i)}-E_{\operatorname{Dr}(i)}
$$

where, ${ }^{E} \operatorname{or}(i)$ is the initial energy possessed by each mobile node of the network. Finally, the Path Residual Energy factor 'PRE' is calculated using residual energy and the initial energy of the nodes along the path as defined in " 7 ".

$$
P R E=\sum_{i=1}^{n} \frac{E_{\operatorname{Re}(i)}}{E_{\operatorname{or}(i)}}
$$

\section{Computation of hop count}

The hop count relates to the number of hops travelled by the packet at a specific point of time when it is initiated from the source node to travel along different links of the network in order to reach the destination. This hop count parameter is gathered by each mobile nodes of the network by incorporating the transmission of specially designated control packet that aids in determining the exact number of intermediate nodes existing between the source and destination of the network. Hop count is the indirect parameter that influences the stability of the path under transmission as its increase leads to greater latency time in the network. Thus, the latency of packet transmission is represented using " 8 " as

$$
P K T_{\text {latency }} \propto \text { Hopcnt }
$$

At this junction, the PAINR need to be moderate, the available energy needs to be high and hop count need to be minimum for portraying path stability under routing.

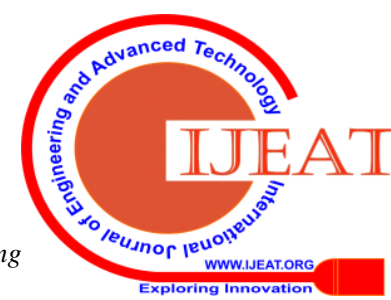




\section{Normalized multi-objective optimization}

The factors considered for investigating path stability is found to be conflicting with one another. These factors of influence cannot be satisfied with low, moderate and high value in a specific instant of routing. Multi-objective optimization is necessary for handling these conflicts of influential parameters responsible for ensuring path stability in the network. Thus, Multi-objective optimization performs the mapping of three factors such as Active interactive neighbor rate, hop count and available energy into a single factor based on weights that determine the enforcement of the factors with constraints and without constraints. The weight inspired sum technique fails to handle the limitations in the integration process as the domination of one impactful parameter may reduce the influence of the other factors under consideration. The method of normalization is proved to eliminate the dominance of one influential factor over the other. Hence lower and upper threshold of normalization technique used for equalizing the impacts of factors that contribute to path stability is represented using " 9 " as

$$
\operatorname{Norm}_{L U T}=\frac{f_{c(i)}-f_{c(\min )}}{f_{c(\text { max })}-f_{c(\min )}}
$$

In this lower and upper threshold of normalization technique, the range of 0 and 1 are considered in order to reduce the dominance of one impactful factor over the other influential factors of path stability.

\section{E. Path Stability Parameter (PSP) Calculation}

The calculation of Path Stability Parameter (PSP) is achieved using " 10 ".

$$
P S P=\frac{P_{\text {(norm) }}}{\text { Hopent }_{\text {(norm) }}}-\text { PAINR }_{(\text {norm) }}
$$

The path with maximum PSP value is optimally chosen for packet forwarding with the possibility that it could provide stable route for data dissemination.

\section{F. Forecasting of path stability using fuzzy logic}

A fuzzy set A is a non-empty set of uncertain data measured by its membership functions defined as " 11 ".

$$
\mu \mathrm{A}(\mathrm{p}): \mathrm{p}->[0,1]
$$

where the values in the range of 0 to 1 represents the membership degree. Fuzzy logic has been developed from fuzzy set. Fuzzy control system is an application of fuzzy logic.

The main objective of using fuzzy logic is to develop a technique to formulate the problem and then find the solution with higher accuracy where there is an uncertainty in the data. There are three modules in Fuzzy control system namely fuzzification, inference engine and defuzzification. Initially crisp inputs are normalized for fuzzification. Then based on the rules of fuzzy inference system the fuzzy output is obtained. Using defuzzification single crisp output is generated from fuzzy output as shown in Fig 1.

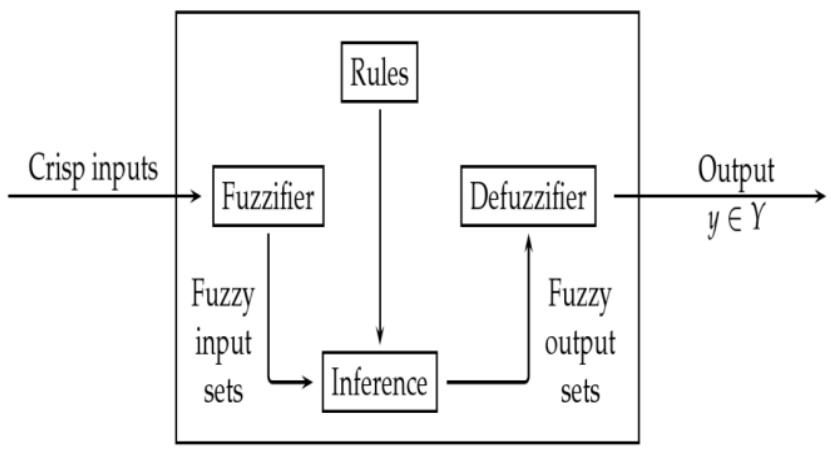

Fig 1. Fuzzy logic system

i) Fuzzification: In this module the input variables are converted into linguistic values to calculate the membership degree. The triangular member ship functions are defined as in " 12 ".

$\mu_{A}(p)=\operatorname{triangular}\left(p, l_{2} m, n\right)=\left\{\begin{array}{cc}0, & p \leq l \\ p-l / m-l_{2} & l \leq p \leq m \\ n-p / n-m, & m \leq p \leq n \\ 0, & n \leq p\end{array}\right.$

The membership functions of PAINR, PRE and Hop cnt are defined as low, medium, high as shown in Fig 2.

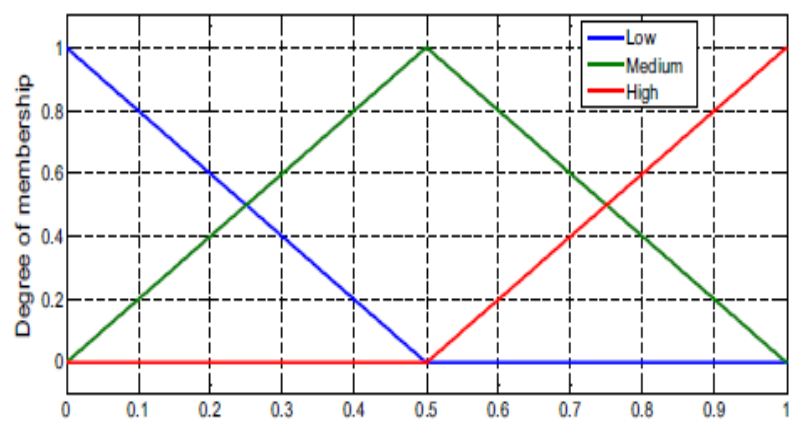

Fig 2. Membership function of PAINR, PRE and Hopcnt

ii) Inference Engine: It calculates the fuzzy output using predefined fuzzy rules. It is Mamdani type system. All inputs are normalized between 0 and 1 before applying them to fuzzy inference system (FIS). There are totally $3 * 3 * 3=27$ rules and the output are classified as Vlow, Rather Low, Low, Low Medium, Medium, High Medium, High, Rather High, Vhigh as shown in Fig 3. Some of the rules are in table I.

iii) Defuzzification: There are many techniques used to convert the fuzzy output from inference engine into crisp value in the defuzzification module. In this paper, defuzzification is done by using the centroid formula as given by

$$
P_{\text {cop }}=\frac{\int \mu_{A}(P)_{p d p}}{\int \mu_{A}(P) d p}
$$


Table I: Some Rules for Fuzzification

\begin{tabular}{|l|l|l|l|}
\hline PRE & PAINR & HOPCNT & PSP \\
\hline Low & Low & Low & Low \\
\hline Low & Low & Medium & Rather Low \\
\hline Low & High & High & VLow \\
\hline Medium & Low & High & L Medium \\
\hline Medium & Low & Medium & High Medium \\
\hline Medium & Medium & Medium & Medium \\
\hline High & Medium & Low & High \\
\hline High & Low & Low & Vhigh \\
\hline High & High & Low & Rather high \\
\hline
\end{tabular}

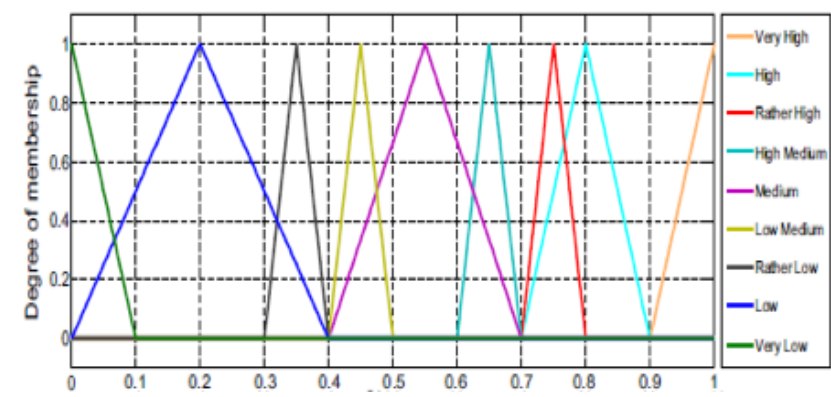

Fig 3. Membership function of path stability

Fig 4 represents the sample screen shot for given input values of PAINR, PRE, hopcnt and fuzzy output for path stability.

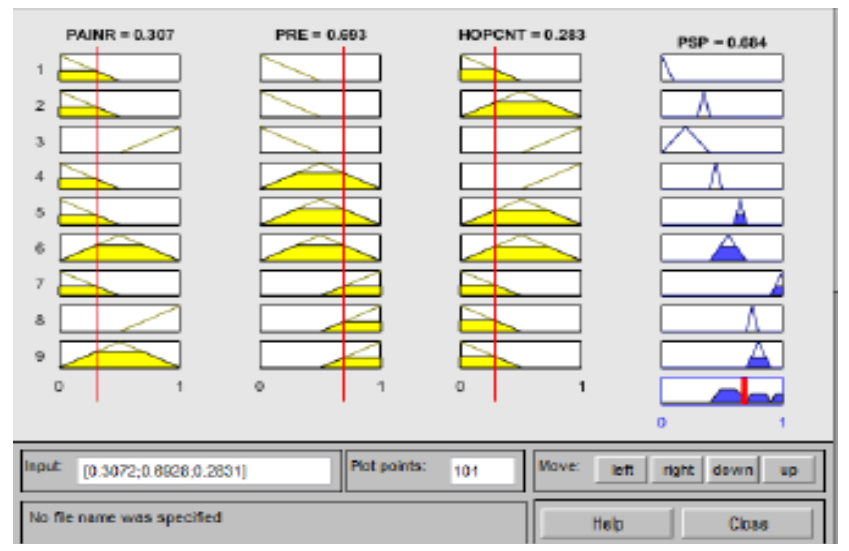

Fig 4. Some fuzzy rules

The following section provides the algorithm used for predicting the path stability using fuzzy logic.

\section{ALGORITHM - FPSFS}

PAINR - Path Active Interactive new Neighbor Rate PRE - Path Residual Energy factor Hop count - No of hops in the path PSP - Path Stability Parameter $\mathrm{n}$ - Number of time slots m - Number of paths

1. Start

2. For each path in each time session ' $\mathrm{t}$ '

3. Calculate PAINR of mobile nodes in the path.

4. Calculate the remaining energy factor PRE of the nodes in the path.

5. Calculate no. of hops in the path.

6. Compute normalized value of PAINR, PRE and hop count.
7. Compute path stability parameter PSP of each path.

8. //Let PSP (1), PSP (2),.., PSP(m) be the PSP for $\mathrm{m}$ no of paths.

9. Data transmission through the Optimal path // In time slot 1

10. Opt $($ path $)=\max (\operatorname{PSP}(1), \operatorname{PSP}(2), \ldots, \operatorname{PSP}(m))$.

11. For each path $\mathrm{p}$ where $1<\mathrm{p}<\mathrm{m} / /$ for time slots 2 to $\mathrm{n}$

12. Calculate the membership function for all the parameters PAINR, PRE, Hop count for each path using Mamdani function.

13. Fuzzify each input using triangular equation.

14. Using fuzzy rules and parameters compute fuzzy output.

15. Defuzzify the fuzzy output to get the crisp output.

16. End for

17. // Let PSP(1), PSP(2),.., PSP(m) be the crisp set of PSP for $m$ no of paths

18. Choose the optimal path from the crisp set of candidate paths for data transmission

19. $\operatorname{Opt}($ path $)=\max (\operatorname{PSP}(1), \operatorname{PSP}(2), \ldots, \operatorname{PSP}(m))$.

20. End For

21. End

In this protocol initially PAINR, PRE and number of hops for each path are calculated and normalized. In the first time slot data transmission is through the optimal path. From second time slot they are given as inputs to fuzzification. Then based on the rules of fuzzy inference system the fuzzy output is obtained. Using defuzzification single crisp output is generated from fuzzy output. The optimal path will be selected with the path which is having maximum PSP value.

\section{SIMULATION RESULTS AND DISCUSSION}

The proposed protocol is evaluated by comparing with various existing protocols. Simulation is focused on different mobility for a fixed no of nodes 100 . NS-2.35 has been used as the simulator to analyze the performance. The simulation is done 5 times and the average values are taken as measurement using Random way point mobility model. Since the nodes are in random motion, the topology of the network will go for indiscriminate change. The simulation area is $1000 * 1000$ square meters. The initial energy level of each node was set to 20 joules. Table 1 presents all the simulation parameters.

Table II: Simulation parameters

\begin{tabular}{|l|l|}
\hline Parameters & Values \\
\hline Simulation tool & NS-2.35 \\
\hline Network nodes & 100 \\
\hline Density of nodes & 15 square meters \\
\hline Transmission Range & 250 meters \\
\hline CBR data rate & $24 \mathrm{Mbps}$ \\
\hline Interval of hello packets & 1 second \\
\hline Mobility speed & $5-50 \mathrm{~m} / \mathrm{sec}$ \\
\hline Traffic type & CBR \\
\hline Radio type & $802.11 \mathrm{a} / \mathrm{g}$ \\
\hline
\end{tabular}

Published By: 
Scenario 1: The mobility of the nodes has been modified from 5 to $50 \mathrm{~m} / \mathrm{s}$ and the performance has been evaluated using various metrics such as Packet delivery ratio, Throughput, Average end to end delay, routing overhead and energy consumption. Since multiple paths are maintained, there is no route rediscovery process when path loss occurs. Hence loss of packets has been reduced which results in increase in the PDR and throughput compare to other existing protocols in terms of $15 \%, 6 \%, 7 \%$ and $33 \%, 18 \%$ and $14 \%$ than AODV [21], Fuzzy-AODV and LB-AOMDV protocols as in Fig 5 and 6.

It is also noted that in terms of congestion, as the proposed protocol gives preference for the node with less PAINR, it reduces the interaction flow between the nodes thus overhead is decreased by $29 \%, 24 \%$ and $17 \%$ and end-to-end delay is reduced by $46 \%, 37 \%$ and $22 \%$ as in Fig 7 and 8 .

As minimum number of neighbor nodes are selected all along the path the amount of energy consumption is also reduced since the over hearing power of the neighbor nodes has been reduced. Energy consumption is decreased by 36\%, $30 \%$ and $16 \%$ than the above-mentioned existing protocols as shown in Fig 9. Table III provides the performance details of various protocols by increasing the speed of mobility of the nodes.

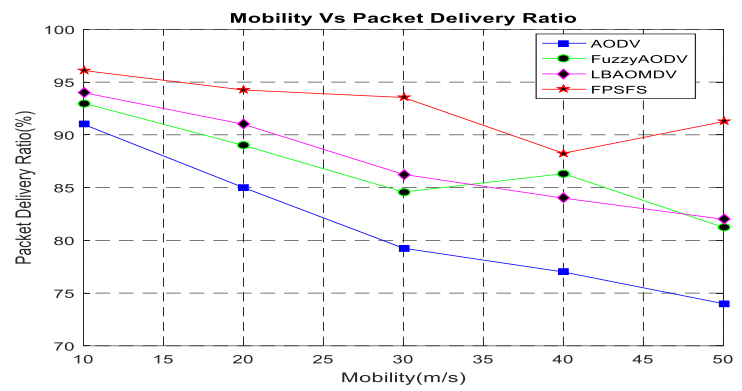

Fig 5. Mobility vs Packet Delivery Ratio

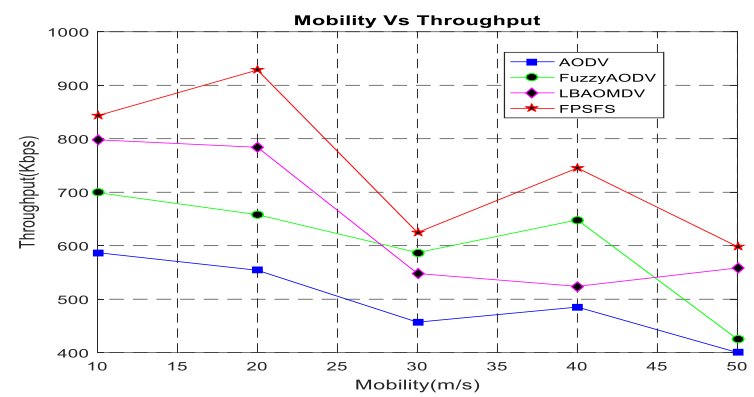

Fig 6. Mobility vs Throughput

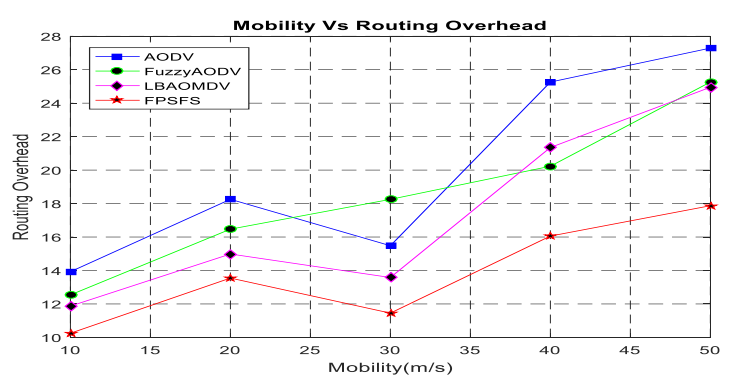

Fig 7. Mobility vs Routing Overhead

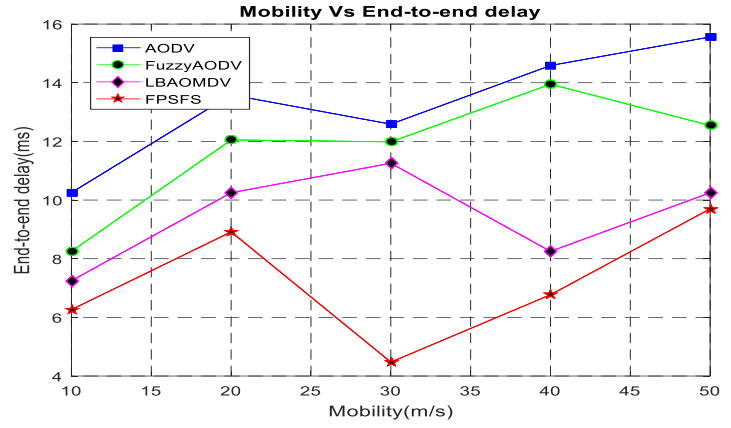

Fig 8. Mobility vs End-to-end-delay

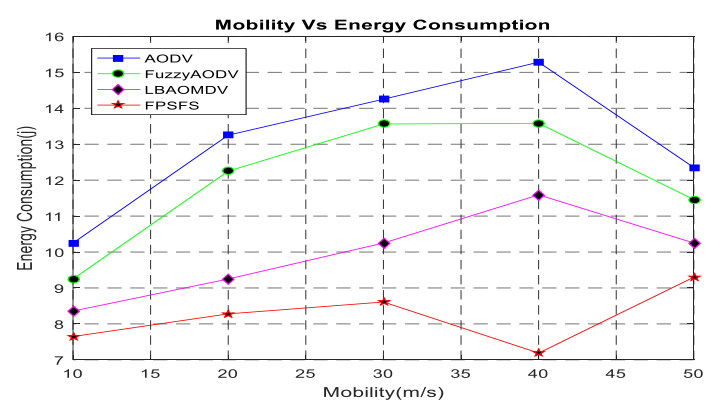

Fig 9. Mobility vs Energy Consumption

Table III: Mobility vs Performance Metrics

\begin{tabular}{|c|c|c|c|c|c|}
\hline & $\begin{array}{l}\text { Mobili } \\
\text { ty }\end{array}$ & AODV & $\begin{array}{l}\text { Fuzzy-A } \\
\text { ODV }\end{array}$ & $\begin{array}{l}\text { LB-AO } \\
\text { MDV }\end{array}$ & FPSFS \\
\hline \multirow{5}{*}{ 竞 } & 10 & 91 & 93 & 94 & 96.1 \\
\hline & 20 & 85 & 89 & 91 & 94.25 \\
\hline & 30 & 79.25 & 84.58 & 86.25 & 93.54 \\
\hline & 40 & 77 & 86.32 & 84 & 88.25 \\
\hline & 50 & 74 & 81.23 & 82 & 91.25 \\
\hline \multirow{5}{*}{ 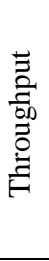 } & 10 & 587 & 699.25 & 798 & 843 \\
\hline & 20 & 554 & 658 & 784 & 928.45 \\
\hline & 30 & 457 & 587 & 548 & 624.58 \\
\hline & 40 & 485.24 & 648.23 & 524 & 745.25 \\
\hline & 50 & 400.25 & 425.12 & 558.4 & 598.25 \\
\hline \multirow{5}{*}{$\frac{\vec{\pi}}{\stackrel{\Xi}{0}}$} & 10 & 10.254 & 8.254 & 7.25 & 6.265 \\
\hline & 20 & 13.587 & 12.05 & 10.25 & 8.914 \\
\hline & 30 & 12.587 & 1.985 & 11.254 & 4.474 \\
\hline & 40 & 14.587 & 13.95 & 8.25 & 6.772 \\
\hline & 50 & 15.56 & 12.54 & 10.25 & 9.701 \\
\hline \multirow{5}{*}{ 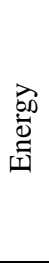 } & 10 & 10.25 & 9.25 & 8.359 & 7.65 \\
\hline & 20 & 13.25 & 12.25 & 9.25 & 8.28 \\
\hline & 30 & 14.25 & 13.56 & 10.25 & 8.61 \\
\hline & 40 & 15.28 & 13.58 & 11.59 & 7.19 \\
\hline & 50 & 12.35 & 11.458 & 10.25 & 9.3 \\
\hline \multirow{3}{*}{. } & 10 & 13.925 & 12.54 & 11.87 & 10.25 \\
\hline & 20 & 18.254 & 16.47 & 14.985 & 13.547 \\
\hline & 30 & 15.48 & 18.25 & 13.58 & 11.452 \\
\hline
\end{tabular}




\begin{tabular}{|l|l|l|l|l|l|}
\hline & 40 & 25.26 & 20.23 & 21.35 & 16.057 \\
\cline { 2 - 6 } & 50 & 27.29 & 25.26 & 24.95 & 17.87 \\
\hline
\end{tabular}

Scenario 2: The number of nodes for the simulation are varied from 10 to 100 in scenario 2. It has been found that increase in the PDF and throughput compare to other existing protocols in terms of $15 \%, 7 \%, 4 \%$ and $44 \%, 17 \%$ and $6 \%$ than AODV [19], Fuzzy-AODV and LB-AOMDV protocols as in Fig 10 and 11. It is also noted that in terms of congestion, as the proposed protocol gives preference for the node with less AINR, it reduces the interaction flow between the nodes thus overhead is decreased by $41 \%, 32 \%$ and $19 \%$ and end-to-end delay is reduced by $55 \%, 43 \%$ and $28 \%$ as shown in Fig 12 and 13. As minimum number of neighbor nodes are selected all along the path the amount of energy consumption is also reduced since the over hearing power of the neighbor nodes has been reduced. From Fig 14 it has been found that energy consumption is decreased by $15 \%, 7 \%$ and $5 \%$ than the above-mentioned existing protocol. Table IV provides the performance details of various protocols by

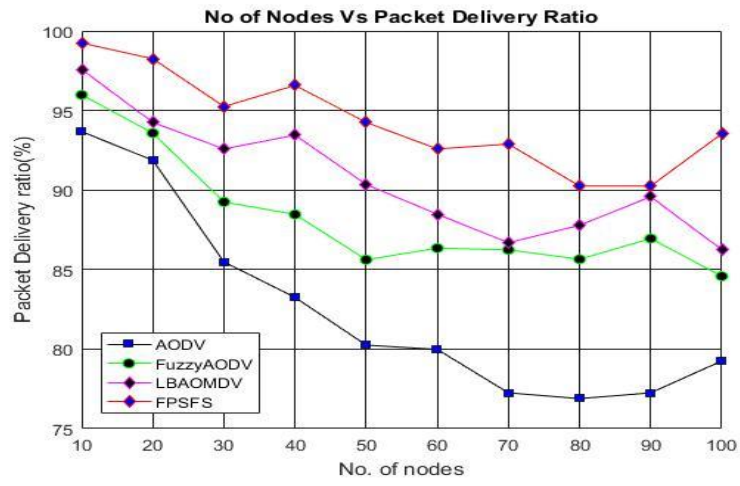

Fig 10. Number of nodes vs Packet Delivery Ratio

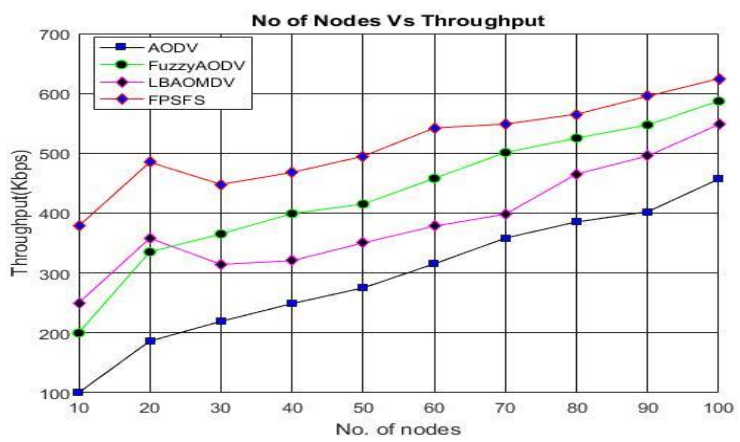

Fig 11. Number of nodes vs Throughput

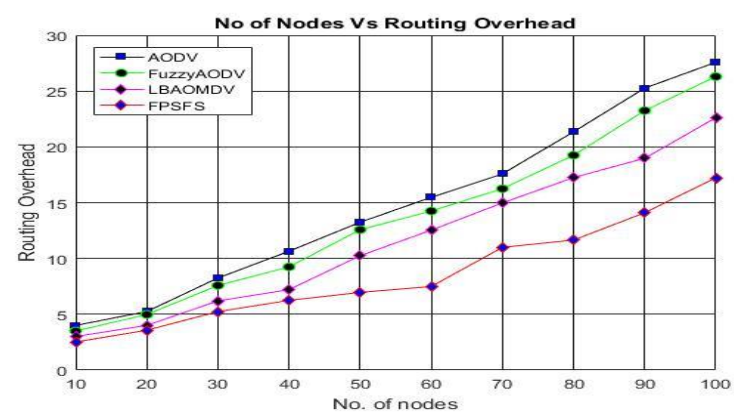

Fig 12. Number of nodes vs Routing overhead increasing the number of nodes.

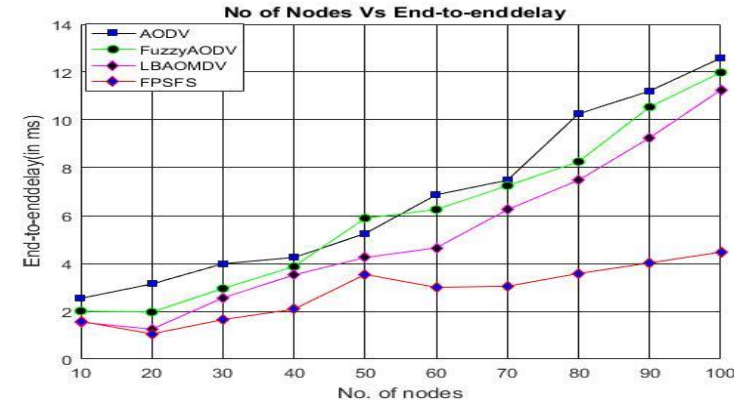

Fig 13. Number of nodes vs End-to-end-delay

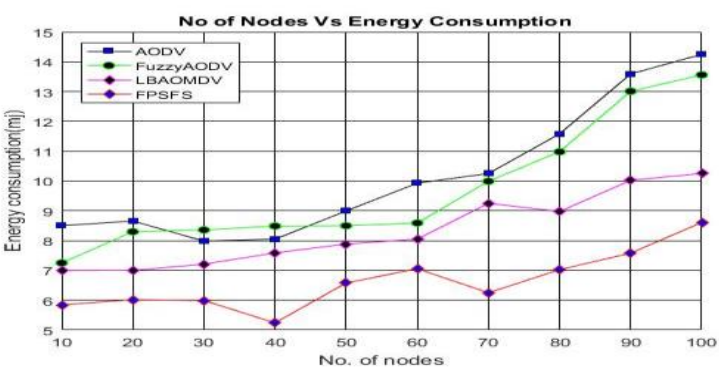

Fig 14. Number of nodes vs Energy consumption

Table IV: Number of nodes vs Performance Metrics

\begin{tabular}{|c|c|c|c|c|c|}
\hline & $\begin{array}{l}\text { No of } \\
\text { nodes }\end{array}$ & AODV & Fuzzy-aodv & Lb-aomdv & Fpsfs \\
\hline \multirow{5}{*}{ 㝓 } & 20 & 91.87 & 93.58 & 94.25 & 98.25 \\
\hline & 40 & 83.25 & 88.47 & 93.45 & 96.58 \\
\hline & 60 & 79.98 & 86.35 & 88.48 & 92.58 \\
\hline & 80 & 76.89 & 85.65 & 87.78 & 90.254 \\
\hline & 100 & 79.256 & 84.58 & 86.25 & 93.541 \\
\hline \multirow{5}{*}{ 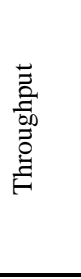 } & 20 & 186.32 & 335.21 & 358 & 485 \\
\hline & 40 & 248.98 & 399.25 & 320.54 & 468 \\
\hline & 60 & 315.24 & 458 & 378.25 & 542.25 \\
\hline & 80 & 385.36 & 525.36 & 465.25 & 565.21 \\
\hline & 100 & 457 & 587 & 548 & 624.58 \\
\hline \multirow{5}{*}{$\frac{\vec{J}}{\stackrel{\Xi}{n}}$} & 20 & 3.14 & 1.965 & 1.25 & 1.054 \\
\hline & 40 & 4.25 & 3.87 & 3.52 & 2.087 \\
\hline & 60 & 6.87 & 6.254 & 4.65 & 2.998 \\
\hline & 80 & 10.254 & 8.254 & 7.48 & 3.58 \\
\hline & 100 & 12.587 & 11.985 & 11.254 & 4.474 \\
\hline \multirow{5}{*}{ 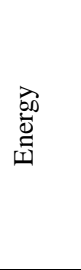 } & 20 & 8.656 & 8.298 & 7 & 6.02 \\
\hline & 40 & 8.047 & 8.487 & 7.587 & 5.24 \\
\hline & 60 & 9.9265 & 8.589 & 8.05 & 7.054 \\
\hline & 80 & 11.58 & 10.98 & 8.974 & 7.024 \\
\hline & 100 & 14.25 & 13.56 & 10.25 & 8.61 \\
\hline בे & 20 & 5.25 & 4.98 & 4.01 & 3.57 \\
\hline
\end{tabular}




\begin{tabular}{|l|l|l|l|l|}
40 & 10.65 & 9.25 & 7.21 & 6.25 \\
\hline 60 & 15.48 & 14.25 & 12.54 & 7.49 \\
\hline 80 & 21.35 & 19.25 & 17.25 & 11.65 \\
\hline 100 & 27.58 & 26.3 & 22.61 & 17.23 \\
\hline
\end{tabular}

Implementation of prediction using fuzzy logic provides multiple path selection. The output of 5 candidate paths given in table $\mathrm{V}$ in which the path with max stability is path $\mathrm{P} 4$. Data transmission will be through P4. If it expires due to some reason then path $\mathrm{P} 2$ will be selected.

Table V : Experimental Results

\begin{tabular}{|c|c|c|c|c|}
\hline \multirow{2}{*}{ PATH } & \multicolumn{3}{|c|}{ FUZZY INPUT } & FUZZY \\
\cline { 2 - 4 } & PRE & PAINR & HOPCNT & OUTPUT \\
\hline P1 & 0.608 & 0.584 & 0.392 & 0.569 \\
\hline P2 & 0.62 & 0.235 & 0.38 & 0.664 \\
\hline P3 & 0.729 & 0.404 & 0.416 & 0.637 \\
\hline P4 & 0.693 & 0.307 & 0.283 & 0.684 \\
\hline P5 & 0.235 & 0.259 & 0.257 & 0.416 \\
\hline
\end{tabular}

\section{CONCLUSION}

In MANET path loss is a major issue due to dynamic nature of the nodes. Hence for effective data communication rapid change of alternate paths is required. Several path prediction mechanisms are prevailing based on various prediction models. Consistent packet delivery is achieved when multiple paths are identified by means of fuzzy logic-based prediction protocol. The simulation study shows that the performance of the new protocol FPSFS provides better results than the existing protocols in terms of routing overhead, energy, throughput, end to end delay and packet delivery ratio. This protocol can be extended by the inclusion of other metrics such as security, RSS and other QOS related factors.

\section{REFERENCES}

1. Boukerche, Azzedine, Begumhan Turgut, Nevin Aydin, Mohammad Z. Ahmad, Ladislau Bölöni, and Damla Turgut, "Routing protocols in ad hoc networks: A survey," Computer networks, vol. 55, no. 13, 2011 pp. 3032-3080.

2. Tran The Son, Hoa Le Minh, Graham Sexton, Nauman Aslam, "Novel encounter-based metric for Mobile Ad Hoc Networks Routing," Journal of Ad Hoc Networks, Elsevier, vol. 14. 2014, pp. 2-14.

3. De Rango, Floriano, Francesca Guerriero, and Peppino Fazio, "Link-stability and energy aware routing protocol in distributed wireless networks," IEEE Transactions on Parallel and Distributed systems, vol. 23, no. 4, 2012, pp. 713-726.

4. Sharma, Varun Kumar, Lal Pratap Verma, and Mahesh Kumar, "A Fuzzy-based Adaptive Energy Efficient Load Distribution Scheme in Ad-hoc Networks," I.J. Intelligent Systems and Applications, vol. 2, 2018, pp. 72-84.

5. Agarwal, Madan Mohan, Anuj Kumar Jhankal, Mahesh Chandra Govil, and Madhavi Sinha. "Fuzzy Logic Controlled Energy Efficient Routing in Mobile Ad-Hoc Networks", International Journal of ComputerNetworks and Wireless Communications, vol. 5, no. 4, 2015, pp. 556-561.

6. Ghazisaidi, Navid, Chadi M. Assi, and Martin Maier. "Intelligent wireless mesh path selection algorithm using fuzzy decision making," Wireless Networks, vol. 18, no. 2, 2012, pp. 129-146.

7. Garg, Harish, and S. P. Sharma. "Fuzzy Multi-objective Optimization of a Synthesis Unit Utilizing Uncertain Data," Journal of Uncertain Systems, vol. 7, no. 1, 2013, pp. 13-21.

8. Smail, Omar, Bernard Cousin, Zoulikha Mekkakia, and Rachida Mekki. "A Multipath Energy-Conserving Routing Protocol for Lifetime Improvement of Wireless Ad Hoc Networks," EURASIP
Journal on Wireless Communications and Networking, Springer Open 2014, no. 139, 2014, pp 1-12.

9. Palaniappan, Senthilnathan, and Kalaiarasan Chellan. "Energy-efficient stable routing using QoS monitoring agents in MANET," EURASIP Journal on Wireless Communications and Networking, vol. 13, no. 12015

10. Singh, Jyoti Prakash, Paramartha Dutta, and Amlan Chakrabarti. "Weighted delay prediction in mobile ad hoc network using fuzzy time series," Egyptian Informatics Journal, vol. 15, no. 2, 2014, pp 105-114.

11. Tabatabaei, Shayesteh, Mohammad Teshnehlab, and Seyed Javad Mirabedini. "Fuzzy-based routing protocol to increase throughput in mobile ad hoc networks," Wireless Personal Communications, vol. 84, no. 4, 2015, pp 2307-2325.

12. Kumaran, R. Senthil. "Implementation of AODV protocol with and without fuzzy logic for reliable multicast routing in ad hoc networks," International Journal of Scientific and Engineering Research, vol. 3, no. 4, 2012, pp. 1-7.

13. Er-rouidi, Mohamed, Houda Moudni, Hassan Faouzi, Hicham Mouncif, and Abdelkrim Merbouha. "A Fuzzy-Based Routing Strategy to Improve Route Stability in MANET Based on AODV," International Conference on Networked Systems, Springer, Cham, 2017, pp. 40-48.

14. Alghamdi, Saleh A. "Load balancing ad hoc on-demand multipath distance vector (LBAOMDV) routing protocol," EURASIP Journal on Wireless Communications and Networking, no. 1, 2015, pp. 242-252.

15. Santhi, G., and Alamelu Nachiappan. "Fuzzy-cost based multiconstrained QoS routing with mobility prediction in MANETs," Egyptian informatics journal, vol.13, no. 1,2012, pp. 19-25.

16. Chettibi, Saloua, and Salim Chikhi, "Dynamic fuzzy logic and reinforcement learning for adaptive energy efficient routing in Mobile Ad hoc Networks," Applied Soft Computing, vol. 38, no. 5, pp. 321-328, 2016.

17. Agarwal, Madan Mohan, Anuj Kumar Jhankal, Mahesh Chandra Govil, and Madhavi Sinha, "Fuzzy Logic Controlled Energy Efficient Routing in Mobile Ad-Hoc Networks," International Journal of Computer Networks and Wireless Communications, vol. 5, no. 4, 2015, pp. 556-561.

18. Pi, Shangchao, and Baolin Sun, "Fuzzy controllers based multipath routing algorithm in MANET," Physics procedia, Elsevier, vol. 24, 2012 pp. 1178-1185.

19. C.Calarany, R.Manoharan, "Path Stability Prediction based on Grey Model (PSPGM) for Reliable Unicast Routing in MANET," jour of adv Research in Dynamical \& Control Systems, vol.10, no. 3, 2018, pp. 675-682

20. Sarkar, Sajal, and Raja Datta. "A secure and energy-efficient stochastic multipath routing for self-organized mobile ad hoc networks," Ad Hoc Networks, vol. 37, 2016, pp. 209-227.

21. Vanaja, K., and Dr R. Umarani. "An analysis of single path aodv vs multipath aomdv on link break using ns-2," International Journal of Electronics and Computer Science Engineering, 2013.

22. [22] Ns2 Simulator.

\section{AUTHORS PROFILE}

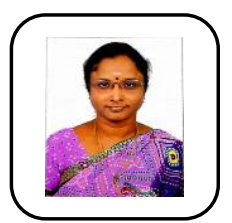

C. Calarany, received her M.C.A degree from Pondicherry Engineering College, Pondicherry University, Pondicherry and M.Phil degree from Manonmaniam Sundaranar University, Thirunelveli in the year 1991 and 2004 respectively. She is working as Assistant Professor in Tagore Govt. Arts and Science College, Pondicherry. She is Pursuing her Ph.D degree in the Department of Computer Science and Engineering Pondicherry Engineering College, Pondicherry. Her area of interests are Mobile Ad-hoc Networks, Network Security and Data Mining.

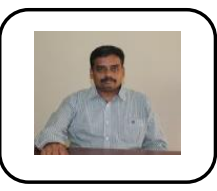

R. Manoharan, is a Professor in the Department of Computer Science and Engineering, Pondicherry Engineering College, Puducherry, India. He received his Master in Technology and Ph.D. from Pondicherry University in the year 1997 and 2007 respectively. His research interests wireless networking, mobile systems, sensor networks, internet technology and Software Engineering. He has published more than 50 Papers in the reputed International journals. 